Gut and Liver, Vol. 10, No. 4, July 2016, pp. 542-548

\title{
Endoscopic Findings of Upper Gastrointestinal Involvement in Primary Vasculitis
}

Eun Jeong Gong ${ }^{1}$, Do Hoon Kim ${ }^{1}$, Joo Hyun Chun ${ }^{1}$, Ji Yong Ahn ${ }^{1}$, Kwi-Sook Choi ${ }^{1}$, Kee Wook Jung ${ }^{1}$, Jeong Hoon Lee ${ }^{1}$, Kee Don Choi ${ }^{1}$, Ho June Song ${ }^{1}$, Gin Hyug Lee ${ }^{1}$, Hwoon-Yong Jung ${ }^{1}$, Jin Ho Kim ${ }^{1}$, In Hye Song ${ }^{2}$, and Yong-Gil Kim ${ }^{3}$

Departments of ${ }^{1}$ Gastroenterology, ${ }^{2}$ Pathology, and ${ }^{3}$ Rheumatology, Asan Medical Center, University of Ulsan College of Medicine, Seoul, Korea

Background/Aims: Gastrointestinal involvement in vasculitis may result in life-threatening complications. However, its variable clinical presentations and endoscopic features, and the rarity of the disease, often result in delayed diagnosis. Methods: Clinical characteristics, endoscopic features, and histopathological findings were reviewed from medical records. Results: Of 6,477 patients with vasculitis, 148 were diagnosed as primary vasculitis with upper gastrointestinal involvement. Of these, 21 cases (14.2\%) were classified as large-vessel vasculitis, 17 cases (11.5\%) as medium-vessel vasculitis, and 110 cases (74.3\%) as small-vessel vasculitis. According to the specific diagnosis, IgA vasculitis (HenochSchönlein purpura) was the most common diagnosis (56.8\%), followed by Takayasu arteritis (14.1\%), microscopic polyangiitis (10.1\%), and polyarteritis nodosa (6.8\%). Gastrointestinal symptoms were present in 113 subjects (76.4\%), with abdominal pain $(78.8 \%)$ the most common symptom. Erosion and ulcers were striking endoscopic features, and the second portion of the duodenum was the most frequently involved site. Biopsy specimens were obtained from 124 patients, and only eight (5.4\%) presented histopathological signs of vasculitis. Conclusions: Diagnosis of vasculitis involving the upper gastrointestinal tract is difficult. Because of the widespread use of endoscopy, combining clinical features with endoscopic findings may facilitate making appropriate diagnoses; however, the diagnostic yield of endoscopic biopsy is low. (Gut Liver 2016;10:542-548)

Key Words: Vasculitis; Gastrointestinal tract; Endoscopy

\section{INTRODUCTION}

Vasculitis is an inflammatory disease of blood vessels, which alter vascular blood flow and can damage dependent organs. ${ }^{1,2}$ The clinical course of vasculitis is variable and depends on the size and type of vessels affected. The clinical manifestations of vasculitis with gastrointestinal involvement range from mild symptoms to life threatening complications such as perforation, acute mesenteric ischemia, and bowel infarction., Therefore, accurate diagnosis and early treatment are mandatory to avoid a poor prognosis, and endoscopic examination helps to identify the site and extent of involvement and assess prognosis.

Diagnosis of gastrointestinal involvement of vasculitis is difficult and often relies on comprehensive investigations including clinical features, laboratory data, radiologic evaluation, and histopathological examination. ${ }^{5-8}$ Suspicion based on clinical manifestations, notably the presence of gastrointestinal symptoms, is important. However, gastrointestinal manifestations are variable, and this can make diagnosis more difficult. In such cases endoscopic examination may be performed to define the extent and nature of the lesion, and endoscopic biopsy might give a clue for diagnosis. We therefore investigated the clinical features of vasculitis with upper gastrointestinal (UGI) involvement, especially from the point of view of endoscopic findings.

\section{MATERIALS AND METHODS}

A total of 6,477 subjects diagnosed as vasculitis at the Asan Medical Center from June 1990 to July 2013 were eligible. Clinical characteristics, endoscopic features, and histopathologic findings were reviewed from medical records.

Primary vasculitis was defined according to the criteria pro-

\footnotetext{
Correspondence to: Do Hoon Kim

Department of Gastroenterology, Asan Medical Center, Asan Digestive Disease Research Institute, University of Ulsan College of Medicine, 88 Olympic-ro 43-gil, Songpa-gu, Seoul 05505, Korea

Tel: +82-2-3010-3193, Fax: +82-2-476-0824, E-mail: dohoon.md@gmail.com

Received on April 6, 2015. Revised on July 5, 2015. Accepted on October 20, 2015. Published online May 26, 2016

pISSN 1976-2283 eISSN 2005-1212 http://dx.doi.org/10.5009/gnl15198

(a) This is an Open Access article distributed under the terms of the Creative Commons Attribution Non-Commercial License (http://creativecommons.org/licenses/by-nc/4.0) which permits unrestricted non-commercial use, distribution, and reproduction in any medium, provided the original work is properly cited.
} 
posed by the American College of Rheumatology and Chapel Hill Consensus Conference nomenclature. ${ }^{9}$ Gastrointestinal involvement of vasculitis was defined as follows: (1) gastrointestinal symptoms that present at the time of the diagnosis of vasculitis and respond to specific therapy for vasculitis; (2) gastrointestinal symptoms that occur during a relapse or flareup of vasculitis and respond to specific therapy for vasculitis; and/or (3) gastrointestinal involvement considered to be associated with vasculitis based on radiologic findings, endoscopic findings, or histopathological examination. Vasculitis is further classified by the size of vessels usually affected; large-sized, medium-sized, or small-sized arteries. Behçet's disease is vasculitis affect vessels of any size and regarded as primary vasculitis rather than vasculitis associated with systemic disease because of the frequency of vasculitis. However, we excluded Behçet's disease in the analyses because there have been several reports regarding the endoscopic features of Behçet's disease. ${ }^{10,11}$

Endoscopy was performed in cases of clinically suspected gastrointestinal tract involvement, and biopsy specimens were taken upon endoscopists' suspicion. Symptoms were categorized as abdominal pain, nausea or vomiting, diarrhea, and gastrointestinal bleeding. This study was approved by the Institutional Review Board of the Asan Medical Center (2014-0255).

Table 1. Clinical Characteristics of Patients with Primary Vasculitis with Upper Gastrointestinal Involvement

\begin{tabular}{lc}
\hline \multicolumn{1}{c}{ Characteristic } & Value \\
\hline Age, yr & $49(13-65)$ \\
Male sex & $73(49.3)$ \\
Classification of vasculitis & \\
Large-vessel vasculitis & \\
$\quad$ Takayasu arteritis & $21(14.1)$ \\
Medium-vessel vasculitis & \\
$\quad$ Polyarteritis nodosa & $10(6.8)$ \\
$\quad$ Kawasaki disease & $7(4.7)$ \\
Small-vessel vasculitis & \\
ANCA-associated vasculitis & $3(2.0)$ \\
Granulomatosis with polyangiitis (Wegener's) & $7(4.7)$ \\
Microscopic polyangiitis & $15(10.1)$ \\
Eosinophilic granulomatosis with polyangiitis & $1(0.7)$ \\
$\quad$ (Churg-Strauss) & \\
IgA vasculitis (Henoch-Schönlein) & $84(56.8)$ \\
Symptoms* & $113(76.3)$ \\
Abdominal pain & $89(78.8)$ \\
Gastrointestinal bleeding & $43(38.1)$ \\
Nausea or vomiting & $18(15.9)$ \\
Diarrhea & $4(3.5)$ \\
\hline
\end{tabular}

Data are presented as median (interquartile range) or number (\%). ANCA, antineutrophil cytoplasmic antibody.

*The sum exceeds 100\% because multiple symptoms were possible.

\section{RESULTS}

\section{Clinical characteristics of patients with vasculitis involving the upper gastrointestinal tract}

During the study period, 912 of 6,477 subjects diagnosed as vasculitis underwent endoscopic examination, and a total of 148 patients were diagnosed as primary vasculitis with UGI involvement. The median age of these 148 patients was 49 years (interquartile range, 13 to 65 years) and the male-to-female ratio was 0.97:1. IgA vasculitis (Henoch-Schönlein purpura [HSP]) was the most common diagnosis $(n=84,56.8 \%)$, followed by Takayasu arteritis $(n=21,14.1 \%)$, microscopic polyangiitis $(n=15$, $10.1 \%)$, and polyarteritis nodosa $(n=10,6.8 \%)$. Three patients died before a specific diagnosis was made; they were classified as antineutrophil cytoplasmic antibody (ANCA)-associated vasculitis which includes granulomatosis with polyangiitis (formerly known as Wegener's granulomatosis), eosinophilic granulomatosis with polyangiitis (formerly Churg-Strauss syndrome). The characteristics of these patients are summarized in Table 1.

Gastrointestinal symptoms were present in 113 of the patients (76.4\%). Abdominal pain was the most common symptom ( $\mathrm{n}=89$, $78.8 \%$ ), and 43 patients (38.1\%) had gastrointestinal bleeding such as melena, hematochezia, and hematemesis. Thirty-five patients (23.6\%) had systemic symptoms including dyspnea, chest pain, headache, or peripheral edema. Symptoms were improved by the treatment for the vasculitis, and some symptoms even resolved without specific therapy, especially in patients with HSP.

\section{Endoscopic findings}

Endoscopic examination was performed in advance to the diagnosis of vasculitis was made in 22 cases (14.9\%). Otherwise, endoscopic examination was performed in patients with clinical features suspicious of vasculitis or patients already diagnosed as vasculitis. Endoscopic findings included erosion, petechiae, nodular change, edema, submucosal hemorrhage, nodularity, and ulcer (Figs 1-3). Since some of these features can be due to abnormalities of mixed nature, more than one finding could be encountered in a single patient. Nonspecific inflammatory changes such as gastritis, esophagitis, and duodenitis were common (77.7\%), however, we did not include these findings as endoscopic manifestations because they are common in the general population and their origin is ill-defined. Erosion and ulcer were striking features of vasculitis, and the second portion of the duodenum and gastric antrum were the most frequent sites involved. Stricture was found in four patients; in three cases in the duodenum, and one case in the jejunum. Endoscopic examination revealed no specific abnormality in six patients. Detailed data on these patients are presented in Table 2.

Biopsy specimens were obtained in 124 subjects. Histopathologic examination of the biopsy specimens revealed signs of vasculitis in only eight patients (5.4\%); seven patients with duodenal involvement of the HSP and one patient with granu- 


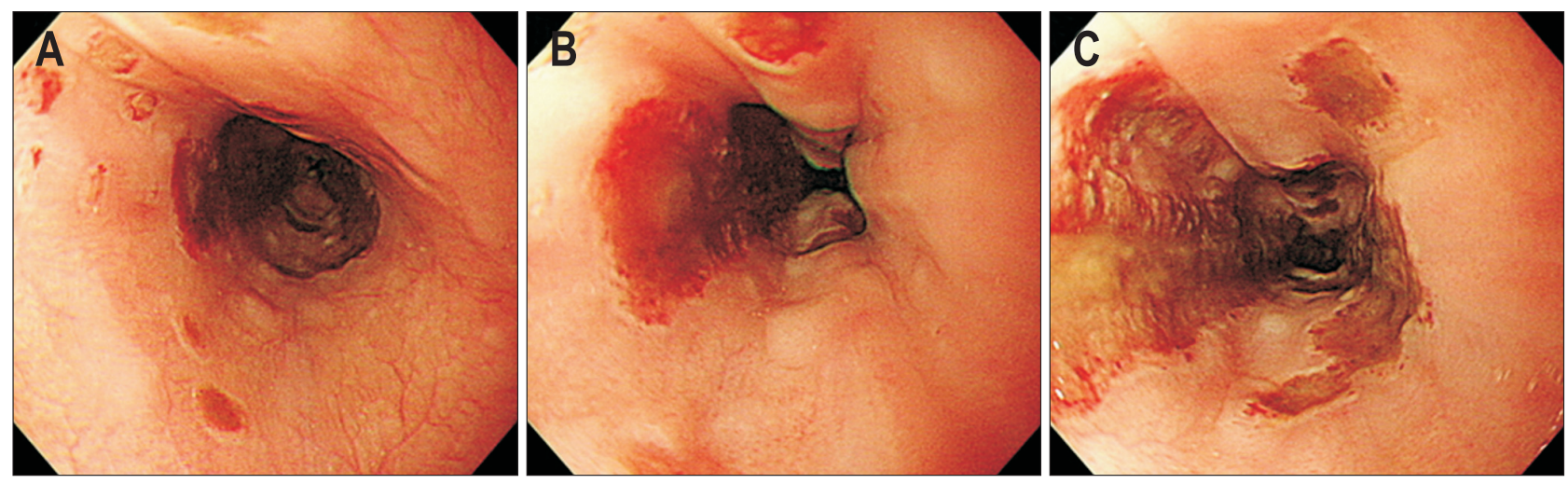

Fig. 1. Endoscopic findings of the esophageal involvement of vasculitis. (A) Erosion and petichia. (B) Submucosal hemorrhage. (C) Multiple, large geographic ulcers on the esophageal body.

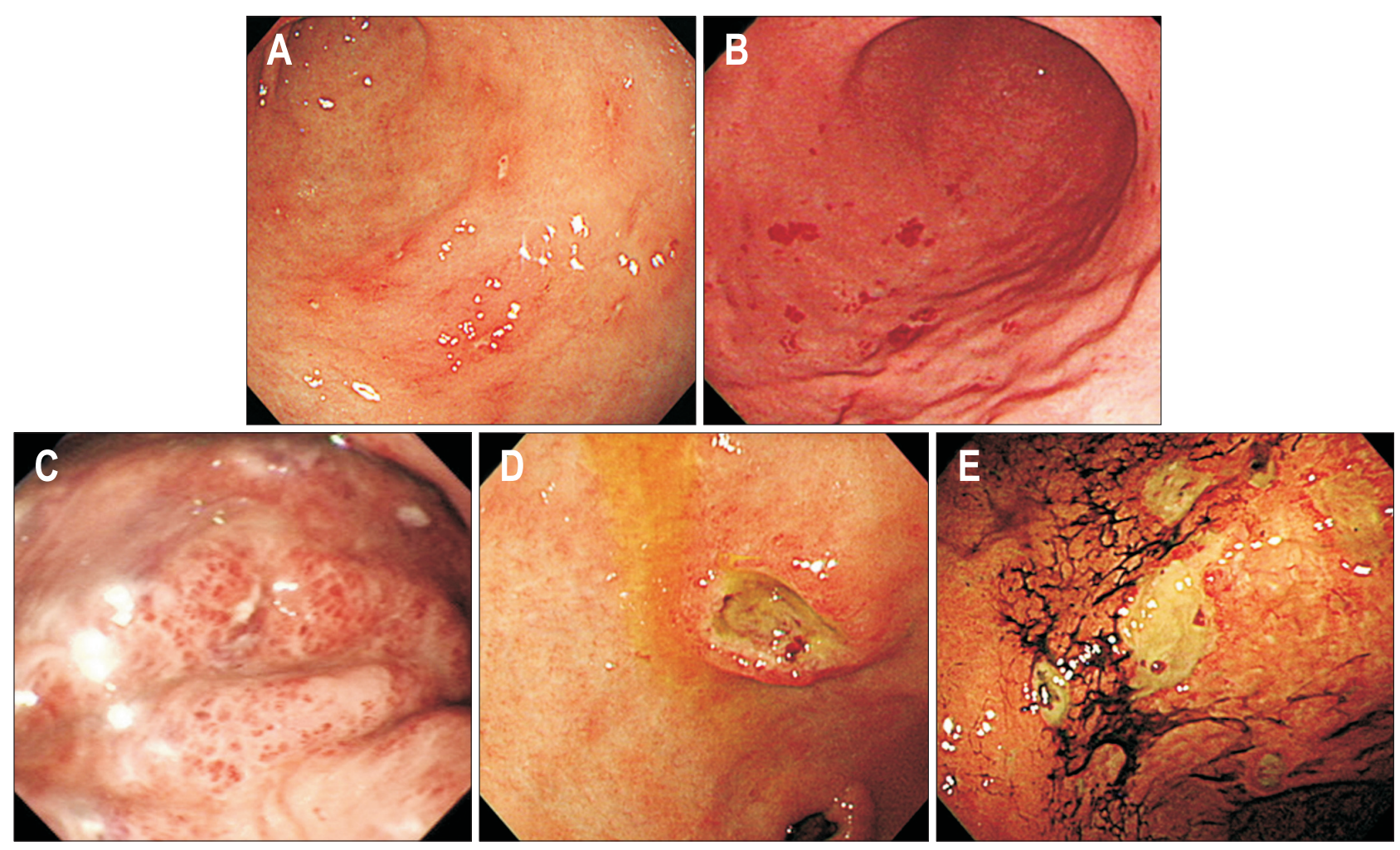

Fig. 2. Endoscopic findings of gastric involvement of vasculitis. (A) Multiple erosions on the antrum. (B) Scattered petechia on the greater curvature of the lower body. (C) Edematous mucosa with submucosal hemorrhage on the greater curvature of the high body. (D) Single deep ulcer with elevated margin on the lesser curvature of the antrum. (E) Multiple ulcers with hematins on the lesser curvature of the lower body.

lomatous angiitis presenting as gastric ulcer (Figs 4 and 5). The median number of biopsy specimens was three in these eight patients. The biopsy specimens of the other patients revealed nonspecific inflammation only.

\section{DISCUSSION}

In the present study, we investigated the clinical features of primary vasculitis with UGI involvement. Of 148 patients, 113 patients (76.4\%) had gastrointestinal symptoms including ab- dominal pain and gastrointestinal bleeding. Endoscopic examination during diagnosis revealed erosion, petechiae, submucosal hemorrhage or ulcers in 142 (95.9\%) of the patients, and the second portion of the duodenum was the site most frequently involved. Endoscopic examination played important role for establishing the diagnosis of vasculitis in only 22 patients (14.9\%) and the diagnostic yield of endoscopic biopsy was low.

Gastrointestinal involvement of vasculitis has been reported variously depending on the diagnosis of vasculitis; 50\% to $85 \%$ for HSP, $14 \%$ to $65 \%$ for polyarteritis nodosa, 3\% to $71 \%$ for 


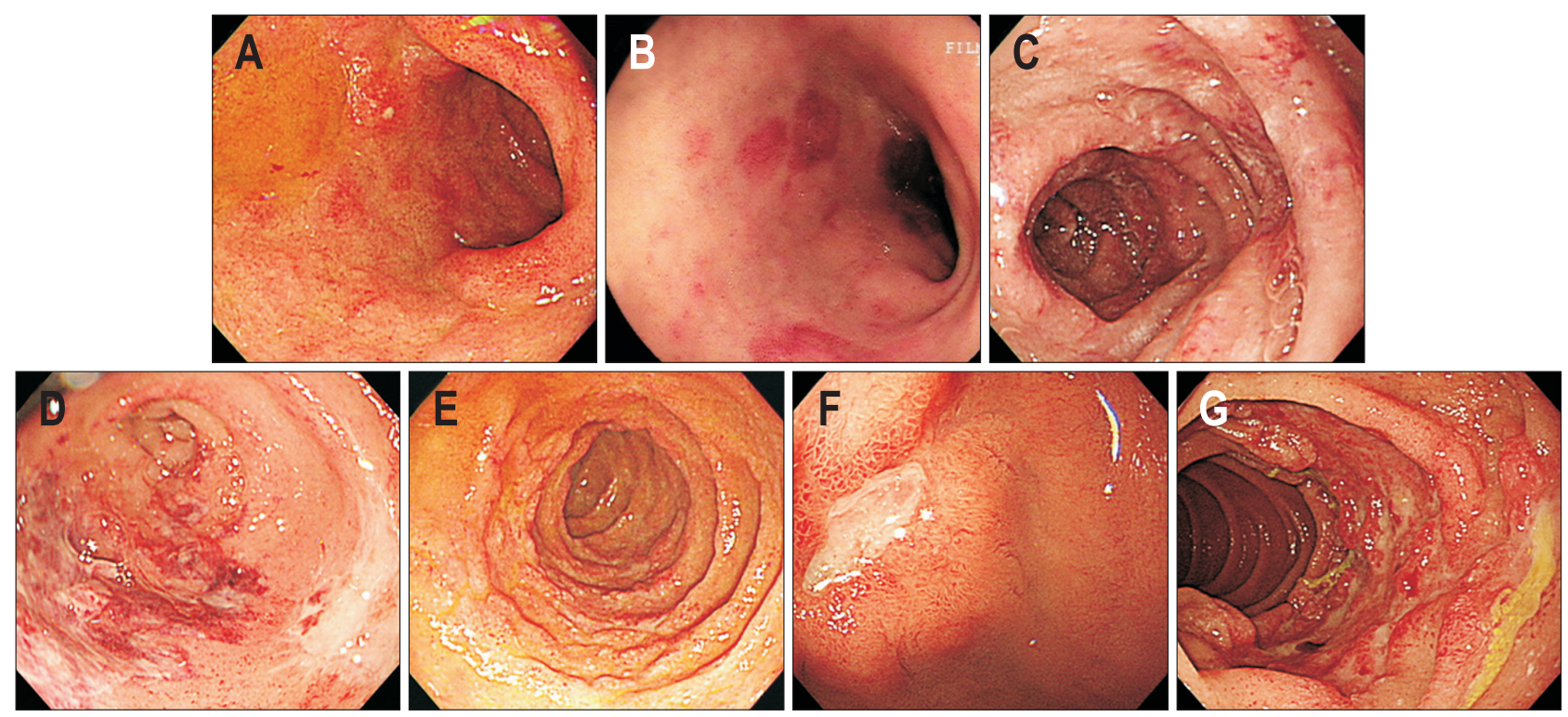

Fig. 3. Endoscopic findings of duodenal involvement of vasculitis. (A) Multiple erosions on the bulb. (B) Scattered petechia on the bulb. (C) Edematous mucosal fold change on the second portion of the duodenum. (D) Submucosal hemorrhage and circular ulcer on the bulb. (E) Diffuse nodular change of the second portion of the duodenum. (F) Single ulcer on the bulb. (G) Multiple circular ulcers with mucosal hyperemia on the second portion of the duodenum.

Table 2. Endoscopic Findings of Vasculitis

\begin{tabular}{|c|c|c|c|c|c|c|c|}
\hline & \multirow{2}{*}{ Erosion } & \multirow{2}{*}{ Petechiae } & \multirow{2}{*}{ Edema } & \multirow{2}{*}{$\begin{array}{l}\text { Submucosal } \\
\text { hemorrhage }\end{array}$} & \multirow{2}{*}{ Nodularity } & \multicolumn{2}{|c|}{ Ulcer } \\
\hline & & & & & & Single & Multiple \\
\hline Esophagus & 1 & 1 & 0 & 1 & 0 & 0 & 6 \\
\hline \multicolumn{8}{|l|}{ Stomach } \\
\hline Antrum & 48 & 9 & 3 & 2 & 0 & 5 & 13 \\
\hline Body & 23 & 9 & 3 & 6 & 0 & 5 & 6 \\
\hline Fundus & 6 & 6 & 2 & 3 & 0 & 0 & 1 \\
\hline \multicolumn{8}{|l|}{ Duodenum } \\
\hline Bulb & 23 & 3 & 4 & 4 & 1 & 6 & 9 \\
\hline 2nd portion & 24 & 5 & 19 & 13 & 9 & 1 & 28 \\
\hline 3rd portion & 4 & 2 & 9 & 6 & 4 & 0 & 12 \\
\hline Jejunum & 0 & 0 & 2 & 0 & 0 & 0 & 2 \\
\hline
\end{tabular}

ANCA-associated vasculitis, and $1 \%$ to $15 \%$ for Takayasu arteritis. $^{12-18}$ Gastrointestinal symptoms were mainly acute abdominal pain, but other symptoms including nausea or vomiting, diarrhea, and gastrointestinal bleeding can be presenting symptoms. ${ }^{14,19-21}$ In addition, gastric outlet obstruction, intussusception, and duodenal necrosis have been reported. ${ }^{22,23}$ In our study, the most common primary vasculitis with UGI involvement was HSP (56.8\%) and 76.4\% of patients with primary vasculitis had gastrointestinal symptoms, abdominal pain being the most common.

The endoscopic findings commonly described in systemic vasculitis are diffuse redness, petechiae, hemorrhagic erosion, nodular change, and ulcer. ${ }^{16,19,24}$ The specific appearance of le- sions and their site preference are important clues that can help differentiate vasculitis from peptic ulcer or drug-induced ulcer. However, the variable clinical presentation and endoscopic features of UGI involvement of systemic vasculitis, and the rarity of the disease, often result in delayed diagnosis. In one report, duodenum and small intestine were the most frequently involved in patients with HSP, and ulcers were rare. ${ }^{13}$ In contrast, in another report, ulcers in the small bowel including duodenum were frequent in these patients. ${ }^{19}$ In our study, the ulcers caused by vasculitis were usually multiple, irregular, and uneven based, and duodenal involvement of the vasculitis usually presented as submucosal hemorrhage, mucosal congestion, and multiple ulcers in the second portion of the duodenum. Recognizing these 

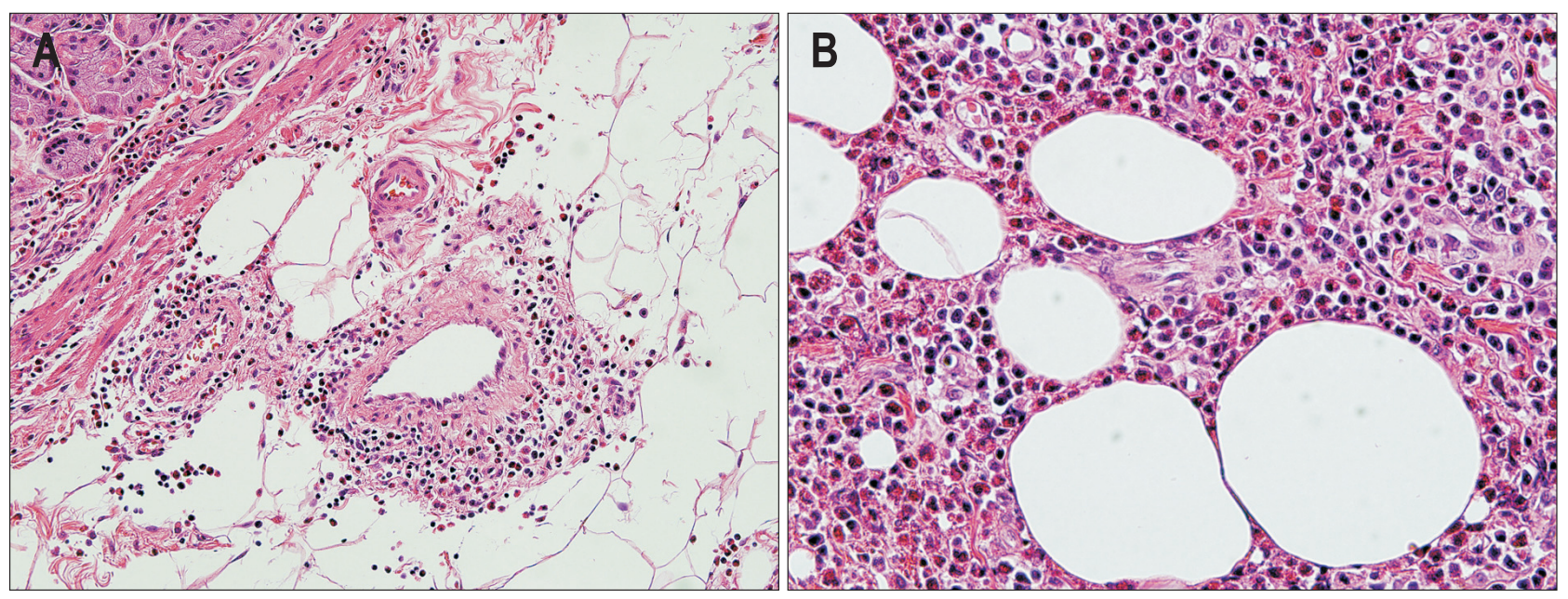

Fig. 4. Representative microscopic images of a small bowel segmental resection specimen of a patient with Henoch-Schönlein purpura. (A) Acute and chronic transmural inflammation with hemorrhage and lymphoid hyperplasia are observed (H\&E stain, $\times 200)$. (B) Neutrophilic infiltration is observed in the small-to-medium sized vessel walls (leukocytoclastic vasculitis) (H\&E stain, $\times 400$ ).
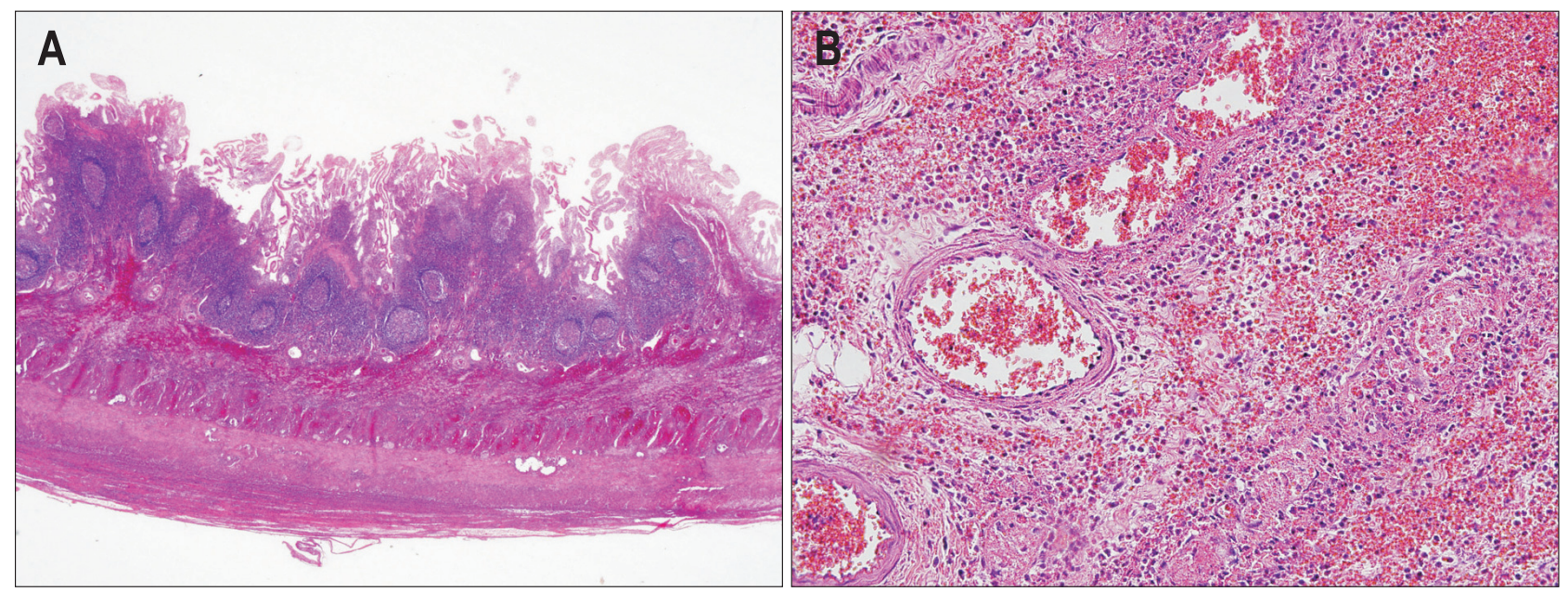

Fig. 5. Pathological findings in a stomach wedge resection specimen of a patient with allergic granulomatous angiitis. (A) Eosinophilic infiltration is evident in small, medium, and large-sized vessels (eosinophilic vasculitis) (H\&E stain, $\times 40)$. (B) There is diffuse and marked eosinophilic infiltration in the gastric wall $(H \& E$ stain, $\times 200)$.

features in such clinical settings may provide clues to the diagnosis of vasculitis. However, the role of endoscopic examination is still limited.

The clinical presentation of gastrointestinal vasculitis may be life-threatening because of complications such as severe ischemia, infarction, and perforation. Gastrointestinal involvement of systemic vasculitis has been reported to be associated with severe complications and poor outcomes especially in patients with polyarteritis nodosa, microscopic angiitis, and granulomatosis with polyangiitis. ${ }^{14,25-28}$ Therefore, accurate diagnosis and early treatment is important to avoid fatal outcomes, and endoscopic examination helps to define the site and extent of involvement and to assess prognosis. However, the diagnosis of vasculitis is difficult and endoscopic findings do not always correlate with presenting symptoms. In addition, endoscopic biopsy has low sensitivity to diagnose vasculitis. ${ }^{3,29,30}$ In one study, biopsy specimens were taken from 23 patients, and none of the UGI biopsies showed evidence of vasculitis whereas lower gastrointestinal tract biopsies revealed signs of vasculitis in three of six patients. ${ }^{21}$ In agreement with these reports, the diagnostic yield of endoscopic biopsy was $5.4 \%$ in this study. This is because most biopsies are limited to the superficial mucosa. ${ }^{10,12,20,31}$ Endoscopic examination could not be used to exclude UGI involvement of primary vasculitis but to exclude other diseases. ${ }^{32}$ In addition, the diagnosis of vasculitis should be made with consideration of all clinical and pathologic information, and neither of these findings should be interpreted in isolation.

Our study has several limitations. First, there may be a selec- 
tion bias because endoscopic examination and endoscopic biopsies were performed upon the decision of the attending physician. In addition, the large proportion of patients were HSP, and this could lead to bias. Second, many factors can be associated with the occurrence of inflammatory changes and ulcers, including Helicobacter pylori infection and the use of nonsteroidal anti-inflammatory drugs. Finally, since the study was retrospective study, most patients did not undergo follow-up endoscopy, and we could not investigate the clinical course of these lesions.

In conclusion, the diagnosis of vasculitis with UGI involvement is difficult. With the wider use of endoscopy, the combination of clinical or radiological features with endoscopic findings may help to make accurate diagnoses; however, the diagnostic yield of endoscopic biopsy is low.

\section{CONFLICTS OF INTEREST}

No potential conflict of interest relevant to this article was reported.

\section{REFERENCES}

1. Babian M, Nasef S, Soloway G. Gastrointestinal infarction as a manifestation of rheumatoid vasculitis. Am J Gastroenterol 1998;93:119-120.

2. Bailey M, Chapin W, Licht H, Reynolds JC. The effects of vasculitis on the gastrointestinal tract and liver. Gastroenterol Clin North Am 1998;27:747-782.

3. Collins DA, Duke 0. Systemic vasculitis presenting with massive bowel infarction. J R Soc Med 1995;88:692-693.

4. Mosley JG, Desai A, Gupta I. Mesenteric arteritis. Gut 1990;31: 956-957.

5. Fraioli P, Barberis M, Rizzato G. Gastrointestinal presentation of Churg Strauss syndrome. Sarcoidosis 1994;11:42-45.

6. Gayraud M, Guillevin L, le Toumelin P, et al. Long-term followup of polyarteritis nodosa, microscopic polyangiitis, and ChurgStrauss syndrome: analysis of four prospective trials including 278 patients. Arthritis Rheum 2001;44:666-675.

7. Généreau T, Lortholary 0, Royer I, Lhote F, Darras-Joly C, Guillevin L. Digestive manifestations of periarteritis nodosa. Gastroenterol Clin Biol 1997;21:503-510.

8. Pinkney JH, Clarke G, Fairclough PD. Gastrointestinal involvement in Wegener's granulomatosis. Gastrointest Endosc 1991;37:411412.

9. Jennette JC, Falk RJ, Bacon PA, et al. 2012 Revised International Chapel Hill Consensus Conference Nomenclature of Vasculitides. Arthritis Rheum 2013;65:1-11.

10. Hokama A, Kishimoto K, Ihama Y, et al. Endoscopic and radiographic features of gastrointestinal involvement in vasculitis. World J Gastrointest Endosc 2012;4:50-56.

11. Ebert EC. Gastrointestinal manifestations of Behçet's disease. Dig Dis Sci 2009;54:201-207.
12. Ahn E, Luk A, Chetty R, Butany J. Vasculitides of the gastrointestinal tract. Semin Diagn Pathol 2009;26:77-88.

13. Esaki M, Matsumoto T, Nakamura S, et al. GI involvement in Henoch-Schönlein purpura. Gastrointest Endosc 2002;56:920-923.

14. Latus J, Koetter I, Fritz P, et al. Gastrointestinal involvement in granulomatosis with polyangiitis and microscopic polyangiitis: histological features and outcome. Int J Rheum Dis 2014;17:412419.

15. Lee EL, Smith HJ, Miller GL 3rd, Burns DK, Weiner H. Ischemic pseudomembranous colitis with perforation due to polyarteritis nodosa. Am J Gastroenterol 1984;79:35-38.

16. Szer IS. Henoch-Schönlein purpura: when and how to treat. J Rheumatol 1996;23:1661-1665.

17. Ebert EC, Hagspiel KD, Nagar M, Schlesinger N. Gastrointestinal involvement in polyarteritis nodosa. Clin Gastroenterol Hepatol 2008;6:960-966.

18. Ha HK, Lee SH, Rha SE, et al. Radiologic features of vasculitis involving the gastrointestinal tract. Radiographics 2000;20:779-794.

19. Nishiyama R, Nakajima N, Ogihara A, et al. Endoscope images of Schönlein-Henoch purpura. Digestion 2008;77:236-241.

20. Zhang Y, Huang X. Gastrointestinal involvement in HenochSchönlein purpura. Scand J Gastroenterol 2008;43:1038-1043.

21. Pagnoux C, Mahr A, Cohen P, Guillevin L. Presentation and outcome of gastrointestinal involvement in systemic necrotizing vasculitides: analysis of 62 patients with polyarteritis nodosa, microscopic polyangiitis, Wegener granulomatosis, Churg-Strauss syndrome, or rheumatoid arthritis-associated vasculitis. Medicine (Baltimore) 2005;84:115-128.

22. Rathore M, Shrivastava R, Goyal R, Radotra BD, Thapa BR. Henoch Schönlein purpura presenting as duodenal ulcer and gastric outlet obstruction. Indian J Pediatr 2014;81:189-190.

23. Becker A, Mader R, Elias M, Lev A, Sayfan J. Duodenal necrosis as the presenting manifestation of polyarteritis nodosa. Clin Rheumatol 2002;21:314-316.

24. Tomomasa T, Hsu JY, Itoh K, Kuroume T. Endoscopic findings in pediatric patients with Henoch-Schonlein purpura and gastrointestinal symptoms. J Pediatr Gastroenterol Nutr 1987;6:725-729.

25. Hiraike Y, Kodaira M, Sano M, et al. Polyarteritis nodosa diagnosed by surgically resected jejunal necrosis following acute abdomen. World J Gastroenterol 2013;19:2830-2834.

26. Shepherd HA, Patel C, Bamforth J, Isaacson P. Upper gastrointestinal endoscopy in systemic vasculitis presenting as an acute abdomen. Endoscopy 1983;15:307-311.

27. Guillevin L, Lhote F, Gayraud M, et al. Prognostic factors in polyarteritis nodosa and Churg-Strauss syndrome: a prospective study in 342 patients. Medicine (Baltimore) 1996;75:17-28.

28. Levine SM, Hellmann DB, Stone JH. Gastrointestinal involvement in polyarteritis nodosa (1986-2000): presentation and outcomes in 24 patients. Am J Med 2002;112:386-391.

29. Wilson RT, Dean PJ, Upshaw JD, Wruble LD. Endoscopic appearance of Wegener's granulomatosis involving the colon. Gastrointest Endosc 1987;33:388-389. 
548 Gut and Liver, Vol. 10, No. 4, July 2016

30. Leen EJ, Rees PJ, Sanderson JD, Wilkinson ML, Filipe MI. A case of Churg-Strauss syndrome: tissue diagnosis established by sigmoidoscopic rectal biopsy. Gut 1996;38:299-301.

31. Ebert EC. Gastrointestinal manifestations of Henoch-Schonlein
Purpura. Dig Dis Sci 2008;53:2011-2019.

32. Novák J, Ottlakán A, Tóth K. Colonic biopsy in Henoch-Schönlein purpura. Gastrointest Endosc 1995;41:519. 